\title{
A comparison of automorphic and Artin L-series of GL(2)-type agreeing at degree one primes
}

\author{
Kimball Martin and Dinakar Ramakrishnan \\ To James Cogdell, with friendship and admiration
}

\section{Introduction}

Let $F$ be a number field and $\rho$ an irreducible Galois representation of Artin type, i.e., $\rho$ is a continuous $\mathbb{C}$-representation of the absolute Galois group $\Gamma_{F}$. Suppose $\pi$ is a cuspidal automorphic representation of $\mathrm{GL}_{n}\left(\mathbb{A}_{F}\right)$ such that the $L$-functions $L(s, \rho)$ and $L(s, \pi)$ agree outside a set $S$ of primes. (Here, these $L$-functions denote Euler products over just the finite primes, so that we may view them as Dirichlet series in a right half plane.) When $S$ is finite, the argument in Theorem 4.6 of DS74 implies these two $L$-functions in fact agree at all places (cf. Appendix A of [Mar04]). We investigate what happens when $S$ is infinite of relative degree $\geq 2$, hence density 0 , for the test case $n=2$. Needless to say, if we already knew how to attach a Galois representation $\rho^{\prime}$ to $\pi$ with $L\left(s, \rho^{\prime}\right)=L(s, \pi)$ (up to a finite number of Euler factors), as is the case when $F$ is totally real and $\pi$ is generated by a Hilbert modular form of weight one (Wil88, [RT83), the desired result would follow immediately from Tchebotarev's theorem, as the Frobenius classes at degree one primes generate the Galois group. Equally, if we knew that $\rho$ is modular attached to a cusp form $\pi^{\prime}$, whose existence is known for $F=\mathbb{Q}$ and $\rho$ odd by Khare-Wintenberger ([Kha10]), then one can compare $\pi$ and $\pi^{\prime}$ using [Ram94]. However, the situation is more complex if $F$ is not totally real or (even for $F$ totally real) if $\rho$ is even. Hopefully, this points to a potential utility of our approach.

We prove the following

TheOREM A. Let $F / k$ be a cyclic extension of number fields of prime degree $p$. Suppose $\rho$ and $\pi$ are as above with their L-functions agreeing at all but a finite number of primes $P$ of $F$ of degree one over $k$. Then $L(s, \rho)=L(s, \pi)$, and moreover, at each place $v, \pi_{v}$ is associated to $\rho_{v}$ according to the local Langlands correspondence. In particular, $\pi$ is tempered, i.e., satisfies the Ramanujan conjecture everywhere, and $L(s, \rho)$ is entire.

The curious aspect of our proof below is that we first prove the temperedness of $\pi$, and then the entireness of $L(s, \rho)$, before concluding the complete equality

2010 Mathematics Subject Classification. Primary 11R39; Secondary 11F70, 11F80.

The first author was partially supported by Simons Collaboration Grant 240605.

The second author was partially supported by NSF grant DMS-1001916. 
of the global $L$-functions. Here are roughly the steps involved. As in the case when $S$ is finite, consider the quotients $L(s, \pi) / L(s, \rho)$ and $L(s, \pi \times \bar{\pi}) / L(s, \rho \otimes \bar{\rho})$, which reduce in $\operatorname{Re}(s)>1$ to a quotient of Euler factors involving (outside finitely many) those of degree $\geq 2$. Then we work over $F^{\prime}=F(\sqrt{-1})$ and, by applying Ram15, we find a suitable $p$-power extension $K$ of $F^{\prime}$ over which even the degree $\geq 2$ Euler factors of the base changes $\pi_{K}$ and $\rho_{K}$ agree (outside finitely many), which furnishes the temperedness of $\pi$. Then we carefully analyze certain degree 8 extensions $K$ of $F$ over which we prove that $L\left(s, \rho_{K}\right)$ is necessarily entire, which is not a priori obvious. In fact we prove this for sufficiently many twists $\rho \otimes \chi$ for unitary characters $\chi$ of $K$. Applying the converse theorem over $K$, we then conclude the existence of an automorphic form ${ }_{K} \Pi$ of $\mathrm{GL}(2) / K$ corresponding to $\rho_{K}$. We then identify $\pi_{K}$ with ${ }_{K} \Pi$. The final step is to descend this correspondence $\rho_{K} \leftrightarrow \pi_{K}$ down to $F$, first down to $F^{\prime}$ (by varying $K / F^{\prime}$ ) and then down to $F$.

The analytic estimate we use for the inverse roots $\alpha_{P}$ of Hecke (for $\pi$ ) is that it is bounded above by a constant times $N(P)^{1 / 4-\delta}$ for a uniform $\delta>0$, and in fact much stronger results are known for GL(2) ([KS02, Kim03, BB11). We need the same estimate (of exponent $1 / 4-\delta$ ) for GL $(n)$ in general to prove even a weaker analogue of our theorem. (There is no difficulty for $n=3$ if $\pi$ is essentially selfdual.) There is a nice estimate for general $n$ due to Luo, Rudnick and Sarnak ([LRS99]), giving the exponent $1-1 / 2 n^{2}$, but this does not suffice for the problem at hand. We can still deduce that for any place $v, L\left(s, \pi_{v}\right)$ has no pole close to $s=0$ to its right, and similarly, we can rule out poles of (the global $L$-function) $L(s, \rho)$ in a thin region in the critical strip.

One can ask the same question more generally for $\ell$-adic representations $\rho_{\ell}$ of $\Gamma_{F}$ satisfying the Fontaine-Mazur conditions, namely that the ramification is confined to a finite number of primes and that $\rho_{\ell}$ is potentially semistable. For the argument of this Note to apply, one would need to know in addition that (i) the Frobenius eigenvalues are pure, i.e., of absolute value $N(P)^{w / 2}$ for a fixed weight $w$ for all but finitely many unramified primes $P$, and (ii) for any finite solvable Galois extension $K / F$, the $L$-function of the restrictions of $\rho_{\ell}$ and $\rho_{\ell} \otimes \rho_{\ell}^{\vee}$ to $\Gamma_{K}$ admit a meromorphic continuation and functional equation of the expected type, which is known in the Artin case by Brauer. When $F$ is totally real, we will need this for at least any compositum $K$ of a totally real (solvable over $F$ ) $K^{\prime}$ with $F(\sqrt{-1}$ ). For $F$ totally real with $\rho_{\ell}$ odd (2-dimensional), crystalline and sufficiently regular relative to $\ell$, these conditions may follow from the potential modularity results of Taylor.

The first author thanks Jim Cogdell for being a wonderful teacher and for his encouragement. The second author would like to thank Jim Cogdell for many years of stimulating mathematical conversations and friendship. We also thank the referee for helpful comments which improved the exposition.

\section{Notations and preliminaries}

Let $F / k$ be a cyclic extension of number fields of prime degree $p, \rho: \Gamma_{F} \rightarrow$ $\mathrm{GL}_{2}(\mathbb{C})$ a continuous, irreducible representation, and $\pi$ a cuspidal automorphic representation of $\mathrm{GL}_{2}\left(\mathbb{A}_{F}\right)$ with central character $\omega_{\pi}$.

Suppose $L\left(s, \rho_{v}\right)=L\left(s, \pi_{v}\right)$ at almost all places $v$ of $F$ of degree 1 over $k$. Then, at $\operatorname{such} v, \operatorname{det}\left(\rho_{v}\right)=\omega_{\pi_{v}}$. Consequently, $\operatorname{det}(\rho)=\omega_{\pi}$ globally. Hence $\omega_{\pi}$ is finite order, so $\pi$ is unitary. 
Now we will recall some basic results which we will use below.

LEMma 1.1 (Landau). Let $L(s)$ be a Dirichlet series with Euler product which converges in some right half-plane. Further suppose that $L(s)$ is of positive type, i.e., that $\log L(s)$ is a Dirichlet series with non-negative coefficients. Let $s_{0}$ be the infimum of all the real $s_{1}$ such that $L(s)$ is holomorphic and non-vanishing in $\operatorname{Re}(s)>s_{1}$. Then, if $s_{0}$ is finite, $L(s)$ has a pole at $s_{0}$, and has no zero to the right of $s_{0}$.

In other words, for such an $L(s)$ of positive type, when we approach $s=0$ on the real line from the right, from a real point of absolute convergence, we will not hit a zero of $L(s)$ until we hit a pole.

We will also need a suitable (weaker) bound towards the Generalized Ramanujan Conjecture for GL(2), which asserts temperedness of $\pi_{v}$ everywhere, i.e., that $L\left(s, \pi_{v}\right)$ has no poles on $\operatorname{Re}(s)>0$ for all $v$. We will need it for any finite solvable extension $K$ of $F$.

THEOREM 1.2. Let $\pi$ be an isobaric automorphic representation of $G L_{2}\left(\mathbb{A}_{K}\right)$, for a number field $K$.

(a) If $\pi$ is (unitary) cuspidal, there exists a $\delta<\frac{1}{4}$ such that, for any place $v$ of $K, L\left(s, \pi_{v}\right)$ has no pole for $\operatorname{Re}(s)>\delta$.

(b) If $\pi$ is an isobaric sum of unitary Hecke characters of $K$, then $\pi$ is tempered, and so for any place $v$ of $K, L\left(s, \pi_{v}\right)$ has no pole for $\operatorname{Re}(s)>0$.

The fact that $L\left(s, \pi_{v}\right)$ has no pole for $\operatorname{Re}(s) \geq \frac{1}{4}$ is originally due to GelbartJacquet GJ78 using Sym ${ }^{2} L$-functions. Subsequently, more precise bounds of $\delta=\frac{1}{9}$ were given by Kim-Shahidi [KS02, and later $\delta=\frac{7}{64}$ by Kim-Sarnak Kim03 and Blomer-Brumley [BB11], using respectively the $\mathrm{Sym}^{3}$ and Sym ${ }^{4}$ $L$-functions of $\pi$.

REMARK 1. Some of the estimates towards the Generalized Ramanujan Conjecture cited above are just stated for $v$ such that $\pi_{v}$ unramified. However, for unitary cuspidal $\pi$ on $\mathrm{GL}(2) / F$, the general case easily reduces to the unramified situation. Indeed, if $\pi_{v}$ is not tempered, we may write it as an irreducible principal series $\pi_{v}=\pi\left(\mu_{1}|\operatorname{det}|^{t}, \mu_{2}|\operatorname{det}|^{-t}\right)$, where $\mu_{1}$ and $\mu_{2}$ are unitary, and $t$ is real and non-zero. Since $\check{\pi}_{v} \simeq \bar{\pi}_{v}$ by unitarity, we see that $\left\{\mu_{1}^{-1}|\operatorname{det}|^{-t}, \mu_{2}^{-1}|\operatorname{det}|^{t}\right\}=$ $\left\{\bar{\mu}_{1}|\operatorname{det}|^{t}, \bar{\mu}_{2}|\operatorname{det}|^{-t}\right\}$. Thus $\mu_{1}^{-1}=\bar{\mu}_{2}=\mu_{2}^{-1}$, i.e., $\mu_{1}=\mu_{2}$. Hence, $\pi_{v}=$ $\pi\left(|\operatorname{det}|^{t},|\operatorname{det}|^{-t}\right) \otimes \mu_{1}$ is just a unitary twist of an unramified principal series. Moreover, as $\mu_{1}$ is a finite order character times $\left.|\cdot|\right|^{i x}$ for some $x \in \mathbb{R}$, we may choose a global unitary character $\lambda$ of (the idele classes of) $F$ such that $\lambda_{v}=\mu_{1}$, and so $\pi \otimes \lambda^{-1}$ is unitary cuspidal with its $v$-component unramified, resulting in a bound for $t$.

The next result we need follows from Lemma 4.5 and the proof of Proposition 5.3 in Ram15.

Proposition 1.3. Fix any $r \geq 1$. There exists a finite solvable extension $K / k$ containing $F$ such that any prime $w$ of $K$ lying over a degree $p$ prime $v$ of $F / k$ has degree $\geq p^{r}$ over $k$.

The three results above will be used to show $\pi$ is tempered. In fact, a more explicit version of Proposition 1.3 for quadratic fields is used in the other two parts 
of the proof as well: showing $L(s, \rho)$ is entire, and showing $\rho$ is modular. For deducing modularity, we will need the following version of the converse theorem à la Jacquet-Langlands for 2-dimensional Galois representations.

Denote by $L^{*}(s, \rho)$ and $L^{*}(s, \pi)$ the completed $L$-functions for $\rho$ and $\chi$.

Theorem 1.4. [JL70, Theorem 12.2] Suppose K/F is a finite Galois extension and $\rho$ is a 2-dimensional representation of the Weil group $W_{K / F}$. If the L-functions $L^{*}(s, \rho \otimes \chi)$ are entire and bounded in vertical strips for all idele class characters $\chi$ of $F$, then $\rho$ is modular.

In our case of $\rho$ being an Artin representation, it follows from a theorem of Brauer that each $L^{*}(s, \rho \otimes \chi)$ is a ratio of entire functions of finite order. Thus knowing $L^{*}(s, \rho \otimes \chi)$ is entire implies it is of finite order, whence bounded in vertical strips. Hence we will only need to check entireness to use this converse theorem.

We remark Booker and Krishnamurthy proved a converse theorem [BK11] requiring only a weaker hypothesis.

We say the global representations $\rho$ and $\pi$ correspond if they do in the sense of the strong Artin conjecture, i.e., that their local $L$-factors agree almost everywhere. For GL(2), we show that this type of correspondence implies the stronger conclusion that we assert in Theorem A, that $\rho_{v}$ and $\pi_{v}$ are associated by the local Langlands correspondence at all $v$. The local Langlands correspondence for GL(2) was established by Kutzko Kut80, and is characterized uniquely by matching of local $L$ and $\epsilon$ - factors of twists by finite-order characters of GL(1) (cf. JL70, Corollary $2.19])$.

Proposition 1.5. Suppose that $L\left(s, \rho_{v}\right)=L\left(s, \pi_{v}\right)$ for almost all $v$. Then $\rho_{v}$ and $\pi_{v}$ correspond in the sense of local Langlands at all $v$ (finite and infinite).

Proof. This is a refinement of the argument in Theorem 4.6 of [DS74] and Mar04, Appendix A].

At a finite place $v$ where $\rho_{v}$ and $\pi_{v}$ are both unramified, this is immediate as $\rho_{v}$ and $\pi_{v}$ are determined by $L\left(s, \rho_{v}\right)$ and $L\left(s, \pi_{v}\right)$. So we only need to show this for $v \mid \infty$ and $v \in S$, where $S$ is the set of nonarchimedean places $v$ at which $\rho$ or $\pi$ is ramified or $L\left(s, \rho_{v}\right) \neq L\left(s, \pi_{v}\right)$.

Observe that, by class field theory, $\operatorname{det} \rho$ corresponds to an idele class character $\omega$ over $F$. From $L\left(s, \rho_{v}\right)=L\left(s, \pi_{v}\right)$, we know $\omega_{v}=\omega_{\pi_{v}}$ for all finite $v \notin S$, and therefore $\omega=\omega_{\pi}$ by Hecke's strong multiplicity one for GL(1). That is to say, $\operatorname{det} \rho$ and $\omega_{\pi}$ correspond via class field theory.

We will use the fact that if $T$ is a finite set of places and $\mu_{v}, v \in T$, are finiteorder characters, there exists a finite-order idele class character $\chi$ globalizing the $\mu_{v}$ 's, i.e., $\chi_{v}=\mu_{v}$ for $v \in T$. This is a standard application of the Grunwald-Wang theorem.

First we establish the local Langlands correspondence for $v \mid \infty$. Choose a finiteorder idele class character $\chi$ which is highly ramified at each $u \in S$ and trivial at each infinite place. Then, for every $u<\infty$, we have $L\left(s, \rho_{u} \otimes \chi_{u}\right)=L\left(s, \pi_{u} \otimes \chi_{u}\right)$. Consequently, comparing the functional equations for $L(s, \rho \otimes \chi)$ and $L(s, \pi \otimes \chi)$ gives

$$
\frac{L_{\infty}(1-s, \bar{\pi})}{L_{\infty}(1-s, \bar{\rho})}=\frac{\epsilon(s, \pi \otimes \chi)}{\epsilon(s, \rho \otimes \chi)} \frac{L_{\infty}(s, \pi)}{L_{\infty}(s, \rho)} .
$$


Since the poles of the $L$-factors on the right hand side of (10) lie in $\operatorname{Re}(s)<\frac{1}{2}$, whereas the poles of the $L$-factors on the left hand side lie in $\operatorname{Re}(s)>\frac{1}{2}$, we can conclude $L_{\infty}(s, \pi)=L_{\infty}(s, \rho)$ as they must have the same poles (cf. [Mar04, Appendix A]).

Note that for $v \mid \infty, \rho_{v}$ must be a direct sum of two characters with are 1 or sgn. If $v$ is complex, of course $\rho_{v}=1 \oplus 1$. Consequently

$$
L_{\infty}(s, \pi)=L_{\infty}(s, \rho)=\Gamma_{\mathbb{R}}(s)^{a} \Gamma_{\mathbb{R}}(s+1)^{b}
$$

for some non-negative integers $a, b$.

We claim that, at any archimedean place $v$, the local factors of $\pi$ and $\rho$ agree. Suppose not. Let $v$ be an errant place, which cannot be complex since the only option is $\Gamma_{\mathbb{C}}(s)^{2}=\Gamma_{\mathbb{R}}(s)^{2} \Gamma_{\mathbb{R}}(s+1)^{2}$ for either factor. So $v$ is real. Since the local factors $L\left(s, \pi_{v}\right)$ are $L\left(s, \rho_{v}\right)$ are different by assumption, at least one of them, say $L\left(s, \pi_{v}\right)$, must be of the form $\Gamma_{\mathbb{R}}(s)^{2}$ or $\Gamma_{\mathbb{R}}(s+1)^{2}$. Call this local factor $G_{1}(s)$, and the other local factor, say $L\left(s, \rho_{v}\right), G_{2}(s)$. Now twist by a finite-order character $\chi$ of $F$ which is sufficiently ramified at the bad finite places in $S$, equals 1 at the archimedean places other than $v$, and at $v$ equals sgn. Then $G_{1}(s)$ (say $\left.L\left(s, \pi_{v} \otimes \operatorname{sgn}\right)\right)$ becomes $G_{1}(s+\delta)$, with $\delta \in\{1,-1\}$. Similarly we see that $G_{2}(s)$ (say $L\left(s, \rho_{v} \otimes \operatorname{sgn}\right)$ ) becomes $G_{2}\left(s+\delta^{\prime}\right)$ with $\delta^{\prime} \in\{1,0,-1\}$, where $\delta^{\prime}=0$ if and only if $G_{2}(s)=\Gamma_{\mathbb{R}}(s) \Gamma_{\mathbb{R}}(s+1)$. The total archimedean identity $L_{\infty}(s, \pi \otimes \chi)=$ $L_{\infty}(s, \rho \otimes \chi)$ persists in this case, and comparing with $L_{\infty}(s, \pi)=L_{\infty}(s, \rho)$ gives $G_{1}(s+\delta) / G_{1}(s)=G_{2}\left(s+\delta^{\prime}\right) / G_{2}(s)$. The only way this can happen is if $G_{1}(s+\delta)=$ $G_{2}\left(s+\delta^{\prime}\right)$, which forces $G_{1}(s)=G_{2}(s)$, contradicting the assumption. Hence the claim.

This in fact implies that $\pi_{v}$ is associated to $\rho_{v}$ by the local Langlands correspondence for $v$ archimedean. Namely, for complex $v, L\left(s, \pi_{v}\right)=\Gamma_{\mathbb{C}}(s)$ and $\pi_{v}$ unitary (or $\omega_{\pi_{v}}=\operatorname{det} \rho_{v}=1$ ) implies $\pi_{v}=\pi(1,1)$. For real $v$, if $\pi_{v}=\pi\left(\mu_{1}, \mu_{2}\right)$ is a unitarizable principal series then $\mu_{i}=\operatorname{sgn}^{m_{i}}|\cdot| s_{i}$ with $\left|s_{i}\right|<\frac{1}{2}$. Hence $L\left(s, \mu_{i}\right)=\Gamma_{\mathbb{R}}(s)$ implies $\mu_{i}=1$ and $L\left(s, \mu_{i}\right)=\Gamma_{\mathbb{R}}(s+1)$ implies $\mu_{i}=$ sgn. Consequently, if $L\left(s, \pi_{v}\right)=\Gamma_{\mathbb{R}}(s)^{c} \Gamma_{\mathbb{R}}(s+1)^{d}$ with $c+d=2$, then $\pi_{v}$ is an isobaric sum of $c$ copies of 1 and $d$ copies of sgn, and therefore matches $\rho_{v}$ in the sense of Langlands.

Finally, consider a finite place $v \in S$. Let $\mu$ be a finite-order character of $F_{v}^{\times}$. Let $\chi$ be an idele class character which is highly ramified at all $u \in S-\{v\}$ such that $\chi_{v}=\mu$. For all $u \notin S$, we have $L\left(s, \pi_{u} \otimes \chi_{u}\right)=L\left(s, \rho_{u} \otimes \chi_{u}\right)$ and $\epsilon\left(s, \pi_{u} \otimes \chi_{u}, \psi_{u}\right)=\epsilon\left(s, \rho_{u} \otimes \chi_{u}, \psi_{u}\right)$ by the local Langlands correspondence. But the same is also true for $u \in S-\{v\}$ by a result of Jacquet and Shalika JS85. which is often called stability of $\gamma$-factors: for twists by sufficiently ramified characters, the $L$-factors are 1 and the $\epsilon$-factors are equal since $\omega_{\pi_{u}}=\operatorname{det}_{\rho_{u}}$. Hence the same comparison of functional equations that led to (1) in the archimedean case gives us

$$
\frac{L\left(1-s, \bar{\pi}_{v} \otimes \bar{\mu}\right)}{L\left(1-s, \bar{\rho}_{v} \otimes \bar{\mu}\right)}=\frac{\epsilon\left(s, \pi_{v} \otimes \mu, \psi_{v}\right)}{\epsilon\left(s, \rho_{v} \otimes \mu, \psi_{v}\right)} \frac{L\left(s, \pi_{v} \otimes \mu\right)}{L\left(s, \rho_{v} \otimes \mu\right)} .
$$

Again, a comparison of the poles implies $L\left(s, \pi_{v} \otimes \mu\right)=L\left(s, \rho_{v} \otimes \mu\right)$, and similarly that $L\left(1-s, \bar{\pi}_{v} \otimes \bar{\mu}\right)=L\left(1-s, \bar{\rho}_{v} \otimes \bar{\mu}\right)$. Consequently, $\epsilon\left(s, \pi_{v} \otimes \mu, \psi_{v}\right)=\epsilon\left(s, \rho_{v} \otimes\right.$ $\left.\mu, \psi_{v}\right)$. Since this is true for all $\mu$, we conclude that $\pi_{v}$ and $\rho_{v}$ must correspond in the sense of local Langlands. 


\section{Temperedness}

To show $\pi$ is tempered, we will make use of solvable base change.

Let $K$ be a solvable extension of $F$. Denote by $\rho_{K}$ the restriction of $\rho$ to $\Gamma_{K}$. Denote by $\pi_{K}$ the base change of $\pi$ to $\mathrm{GL}_{2}\left(\mathbb{A}_{K}\right)$, whose automorphy we know by Langlands ([Lan80 $]$ ). More precisely, $\pi_{K}$ is either cuspidal or else an isobaric sum of two unitary Hecke characters of $K$.

Put

$$
\Lambda_{K}(s)=\frac{L^{*}\left(s, \pi_{K} \times \bar{\pi}_{K}\right)}{L^{*}\left(s, \rho_{K} \times \bar{\rho}_{K}\right)} .
$$

If there is no confusion, we will write $\Lambda$ instead of $\Lambda_{K}$. This has a factorization,

$$
\Lambda(s)=\prod_{v} \Lambda_{v}(s), \quad \text { where } \Lambda_{v}(s)=\frac{L\left(s, \pi_{K, v} \times \bar{\pi}_{K, v}\right)}{L\left(s, \rho_{K, v} \times \bar{\rho}_{K, v}\right)},
$$

for any place $v$ of $K$, and is a priori analytic with no zeroes for $\operatorname{Re}(s)>1$. What is crucial for us is that the logarithms of the numerator and denominator of the non-archimedean part of $\Lambda$ are Dirichlet series with positive coefficients, so we will be able to apply Landau's lemma.

For an arbitrary set $S$ of places of $K$, we write $\Lambda_{S}(s)=\prod_{v \in S} \Lambda_{v}(s)$. Denote by $S_{j}$ the set of finite places $v$ of $K$ of degree $j$ over $k$ for which $\rho_{K}, \pi_{K}$ and $K$ are unramified, but $\rho_{K, v}$ and $\pi_{K, v}$ do not correspond. Then we can write

$$
\Lambda(s)=\Lambda_{\Sigma}(s) \prod_{j \geq 2} \Lambda_{S_{j}}(s),
$$

where $\Sigma$ is a finite set containing $S_{1}$, the archimedean places, and the set of finite places where $\pi, \rho$ or $F$ is ramified.

Then $\Lambda(s)$ satisfies a functional equation of the form

$$
\Lambda(s)=\epsilon(s) \Lambda(1-s),
$$

where $\epsilon(s)$ is an invertible holomorphic function on $\mathbb{C}$.

To show $\pi$ is tempered, we will use the following lemma in two different places.

Lemma 2.1. Let $\delta$ be as in Theorem 1.2, and $S_{j}$ as above for $K=F$. Then

(i) $L_{S_{j}}(s, \pi \times \bar{\pi})$ has no poles or zeroes on $\operatorname{Re}(s)>\frac{1}{j}+2 \delta$; and

(ii) $L_{S_{j}}(s, \rho \times \bar{\rho})$ has no poles or zeroes on $\operatorname{Re}(s)>\frac{1}{j}$.

Proof. Let us prove (i).

Suppose $v \in S_{j}$. Let $\alpha_{1, v}$ and $\alpha_{2, v}$ be the Satake parameters for $\pi_{v}$ and $\beta_{v}=\max \left\{\left|\alpha_{1, v}\right|,\left|\alpha_{2, v}\right|\right\}$. Then

(6)

$$
\log L\left(s, \pi_{v} \times \bar{\pi}_{v}\right) \leq \sum_{i=1}^{2} \sum_{l=1}^{2} \sum_{m \geq 1} \frac{\left(\alpha_{i, v} \bar{\alpha}_{l, v}\right)^{m}}{m q_{v}^{m s}} \leq 4 \sum_{m \geq 1}\left(\frac{\beta_{v}^{2}}{q_{v}^{s}}\right)^{m} \leq 4 \sum_{m \geq 1} \frac{1}{q_{v}^{(s-2 \delta) m}},
$$

where the last step follows from the bound in Theorem 1.2, which is equivalent to $\beta_{v}<q_{v}^{\delta}$. In particular, the local factor $L\left(s, \pi_{v} \times \bar{\pi}_{v}\right)$ (which is never zero) is holomorphic for $\operatorname{Re}(s)>2 \delta$. 
Let $p_{v}^{f_{v}}$ denote the norm of the prime of $k$ below $v$, where $p_{v}$ is a rational prime. Then from (6), we see

$$
\begin{aligned}
\log L_{S_{j}}(s, \pi \times \bar{\pi}) & \leq 4 \sum_{v \in S_{j}} \sum_{m \geq 1} \frac{1}{p_{v}^{f_{v} j(s-2 / 9) m}} \leq 4 \sum_{v \in S_{j}} \sum_{m \geq 1} \frac{1}{p_{v}^{j(s-2 / 9) m}} \\
& \leq 4[k: \mathbb{Q}] \sum_{p_{i}} \sum_{m \geq 1} \frac{1}{\left(p_{i}^{m}\right)^{j(s-2 / 9)}} \leq 4 \sum_{n \geq 1} \frac{1}{n^{j(s-2 / 9)}},
\end{aligned}
$$

where $p_{i}$ runs over all primes in the penultimate inequality. This series converges absolutely, and uniformly in compact subsets of the region of $s \in \mathbb{C}$ with

$$
\operatorname{Re}(s)>\frac{1}{j}+2 \delta
$$

Since $L_{S_{j}}(s, \pi \times \bar{\pi})$ is of positive type, we may apply Landau's lemma (Lemma 1.1) to conclude that it is also non-zero and holomorphic for $\operatorname{Re}(s)>\frac{1}{j}+2 \delta$, which implies (i). (Since this incomplete $L$-function has infinitely many Euler factors, it is not obvious that it is non-zero in this region without applying Landau.)

The argument for (ii) is the same, except that one uses the fact that the Frobenius eigenvalues for $\rho_{v}$ lie on $\operatorname{Re}(s)=0$ in place of Theorem 1.2 .

Proposition 2.2. For each place $v$ of $F, \pi_{v}$ is tempered.

Proof. By Theorem 1.2, for any finite set $\Sigma, \Lambda_{\Sigma}$ has no poles for $\operatorname{Re}(s) \geq \frac{1}{2}$.

Fix $N$ such that $N>\left(\frac{1}{2}-2 \delta\right)^{-1}$. Then Lemma 2.1 implies that $\Lambda_{S_{j}}(s)$ has no poles (or zeroes) in the region $\operatorname{Re}(s) \geq \frac{1}{2}$ for $j \geq N$. Fix $r$ such that $p^{r} \geq N$. By Proposition 1.3. there is a solvable $K / F$ such that each prime $v$ of degree $p$ in $F / k$ splits into primes all of degree $\geq p^{r}$ in $K / k$. Then for all but finitely many primes $v$ of degree $<N$ in $K / k$, we have $\Lambda_{K, v}(s)=1$, so in fact we can rewrite (4) as

$$
\Lambda_{K}(s)=\Lambda_{K, \Sigma}(s) \prod_{j \geq N} \Lambda_{K, S_{j}}(s),
$$

for a finite set $\Sigma$.

Hence Lemma 2.1 implies $\Lambda_{K}$ has no zeroes or poles for $\operatorname{Re}(s) \geq \frac{1}{2}$. Therefore, by the functional equation (5), $\Lambda_{K}$ is entire and nowhere zero.

Now suppose $\pi_{v}$ is non-tempered for some place $v$ of $F$. Then $\pi_{v}$ is an irreducible principal series $\pi_{v}=\pi\left(\mu_{1}, \mu_{2}\right)$ and $L\left(s, \pi_{v}\right)=L\left(s, \mu_{1}\right) L\left(s, \mu_{2}\right)$, so $L\left(s, \pi_{v} \times \bar{\pi}_{v}\right)=\prod_{i=1}^{2} \prod_{j=1}^{2} L\left(s, \mu_{i} \bar{\mu}_{j}\right)$.

Consider first $v<\infty$. Write $L\left(s, \mu_{i}\right)=\left(1-\alpha_{i, v} q_{v}^{-s}\right)^{-1}$ for $i=1,2$. Interchanging $\alpha_{1,2}$ and $\alpha_{2, v}$ if necessary, we may assume $\left|\alpha_{1, v}\right|>1$. Then for any place $w$ of $K$ above $v, L\left(s, \pi_{K, w}\right)^{-1}$ has a factor of the form $\left(1-\alpha_{1, v}^{f_{w}} q_{w}^{-s}\right)$ where $f_{w}=\left[K_{w}: F_{v}\right]$. Hence $L\left(s, \pi_{K, w} \times \bar{\pi}_{K, w}\right)^{-1}$ has $\left(1-\left|\alpha_{1, v}\right|^{2 f_{w}} q_{w}^{-s}\right)$ as a factor. Looking at the Dirichlet series $\log L\left(s, \pi_{K, w} \times \bar{\pi}_{K, w}\right)$, Landau's lemma tells us $L\left(s, \pi_{K, w} \times \bar{\pi}_{K, w}\right)$ has a pole at $s_{0}=\frac{2 \log \left|\alpha_{1, v}\right|}{\log q_{v}}$.

Now consider $v \mid \infty$. We may assume $L\left(s, \mu_{1}\right)$ has a pole to the right of $\operatorname{Re}(s)=0$. Again suppose $w$ is a place of $K$ with $w \mid v$. Either $L\left(s, \pi_{K, w} \times \bar{\pi}_{K, w}\right)$ is $L\left(s, \mu_{1} \bar{\mu}_{1}\right)$ (when $v$ splits in $K$ ) or it equals $L\left(s, \mu_{1, \mathbb{C}} \bar{\mu}_{1, \mathbb{C}}\right)$, where $\mu_{1, \mathbb{C}}(z)=\mu_{1}(z \bar{z})$. If we choose $s_{0}>0$ so that both $L\left(s, \mu_{1} \bar{\mu}_{1}\right)$ and $L\left(s, \mu_{1, \mathbb{C}} \bar{\mu}_{1, \mathbb{C}}\right)$ have poles in $\operatorname{Re}(s) \geq s_{0}$, then the same is true for $L\left(s, \pi_{K, w} \times \bar{\pi}_{K, w}\right)$.

Hence, in either case $v<\infty$ or $v \mid \infty$, there exists $s_{0}>0$, independent of $K$, such that $L\left(s, \pi_{K, w} \times \bar{\pi}_{K, w}\right)$ has a pole in $\operatorname{Re}(s) \geq s_{0}$. Another application of Landau's 
lemma tells us $L_{\Sigma}\left(s, \pi_{K} \times \bar{\pi}_{K}\right) \prod_{j \geq N} L_{S_{j}}\left(s, \pi_{K} \times \bar{\pi}_{K}\right)$ must also have a pole in $\operatorname{Re}(s) \geq s_{0}$.

Since $\Lambda_{K}$ is entire, it must be that the denominator $L_{\Sigma}\left(s, \rho_{K} \times \bar{\rho}_{K}\right) \prod_{j \geq N} L_{S_{j}}\left(s, \rho_{K} \times\right.$ $\left.\bar{\rho}_{K}\right)$ of $\Lambda_{K}(s)$ also has a pole at $s_{0}$. However, $L_{\Sigma}\left(s, \rho_{K} \times \bar{\rho}_{K}\right)$ has no poles to the right of $\operatorname{Re}(s)=0$, and by Lemma 2.1, $L_{S_{j}}\left(s, \rho_{K} \times \bar{\rho}_{K}\right)$ has no poles to the right of $\operatorname{Re}(s)=\frac{1}{j}$. Hence $s_{0} \leq \frac{1}{j}$.

Take $r_{0} \geq r$ such that $p^{r_{0}}>\frac{1}{s_{0}}$. By Proposition 1.3, we may replace $K$ by a larger solvable extension so that every prime of degree $p$ in $F$ splits into primes of degree at least $p^{r_{0}}$ in $K$. But then the denominator of $\Lambda_{K}(s)$ has no poles to the right of $p^{-r_{0}}<s_{0}$, a contradiction.

\section{Entireness}

Now we would like to deduce that $L(s, \rho)$ is entire. However, we cannot directly do this when $F / k$ is quadratic, but only over a quadratic or biquadratic extension $K$. Consequently we deduce that $\rho$ and $\pi$ correspond over $K$. In the final section, we will deduce that $\rho$ and $\pi$ correspond over $F$, which will imply the entireness of $L(s, \rho)$. From here on, $i$ will denote a primitive fourth root of 1 in $\bar{F}$.

Proposition 3.1. There exists a solvable extension $K / k$ containing $F$, depending only on $F / k$, such that $L^{*}\left(s, \rho_{K}\right)$ is entire. In fact, if $p=[F: k]>2$, we can take $K=F$. If $p=2$ and $i \in F$, then we can take $K / k$ to be cyclic of degree 4. If $p=2$ and $i \notin F$, then we can take $K / k$ so that $K / F$ is biquadratic.

We will actually construct the field $K$ in the proof, and the explicit construction will be used in the next section to prove our main theorem.

Proof. Consider the ratio of $L$-functions

$$
\mathcal{L}_{K}(s)=\frac{L^{*}\left(s, \rho_{K}\right)}{L^{*}\left(s, \pi_{K}\right)}
$$

which is analytic for $\operatorname{Re}(s)>1$. Since $L\left(s, \pi_{K}\right)$ has no poles, to show $L^{*}\left(s, \rho_{K}\right)$ is entire, it suffices to show $\mathcal{L}_{K}(s)$ has no poles.

As with $\Lambda$, we may write $\mathcal{L}_{K}=\prod_{v} \mathcal{L}_{K, v}$ and define $\mathcal{L}_{K, S}=\prod_{v \in S} \mathcal{L}_{K, v}$. Then note that we can write

$$
\mathcal{L}_{K}(s)=\mathcal{L}_{K, \Sigma}(s) \prod_{j>1} \frac{L_{S_{j}}\left(s, \rho_{K}\right)}{L_{S_{j}}\left(s, \pi_{K}\right)}
$$

for some finite set $\Sigma$. As with $\Lambda$, it satisfies a functional equation of the form

$$
\mathcal{L}_{K}(s)=\epsilon_{\mathcal{L}, K}(s) \mathcal{L}_{K}(1-s),
$$

with $\epsilon_{\mathcal{L}, K}(s)$ everywhere invertible. Hence it suffices to show $\mathcal{L}_{K}(s)$ is analytic in $\operatorname{Re}(s) \geq \frac{1}{2}$.

Note $\mathcal{L}_{K, \Sigma}(s)$ is analytic for $\operatorname{Re}(s) \geq \frac{1}{2}$ (in fact for $\operatorname{Re}(s)>0$, since $\pi$ must be tempered). The same argument as in Lemma 2.1 shows that $L_{S_{j}}\left(s, \rho_{K}\right)$ and $L_{S_{j}}\left(s, \pi_{K}\right)$ are both holomorphic and never zero in $\operatorname{Re}(s)>\frac{1}{j}$, using the fact that now we know $\pi$ is tempered (Proposition 2.2). Hence if $p>2$, then, already for $K=F$, (10) implies that $\mathcal{L}_{K}(s)$ is analytic in $\operatorname{Re}(s)>\frac{1}{p}$, and we are done.

Now suppose $p=2$. Here we use a more explicit version of Proposition 1.3 for cyclic $p^{2}$-extensions: if $K \supset F \supset k$ is a chain of cyclic $p$-extensions with $K / k$ cyclic, 
then every unramified inert prime $v$ in $F / k$ lies under a (unique) degree $p^{2}$ prime $w$ in $K / k$ [Ram15, Lemma 4.4].

Write $F=k(\sqrt{\alpha})$ with $\alpha \in k$. If $i \in F$, then $K=k\left(\alpha^{1 / 4}\right)$ is a cyclic extension of degree 4 . Hence the lemma just quoted means that $\mathcal{L}_{K}(s)$ is analytic in $\operatorname{Re}(s)>\frac{1}{4}$ and we are done.

We may therefore assume $i \notin F$. Put $F_{1}=k(i)$ and $F_{2}=k(\sqrt{-\alpha})$. Let $E=k(i, \sqrt{\alpha})=F(i)$ be the compositum of these fields, which is biquadratic over $k$. For $i=1,2$, let $\alpha_{i} \in F_{i}$ such that $E=F_{i}\left(\sqrt{\alpha_{i}}\right)$, and put $K_{i}=F_{i}\left(\alpha_{i}^{1 / 4}\right)$. Then $K_{i} / F_{i}$ is cyclic of degree 4 with with $E$ as the intermediate subfield $(i=1,2)$. Denote by $K$ the compositum $K_{1} K_{2}$. (This construction of $K$ is the $p=2$ case of a construction given in Ram15, Section 5].) Here is a diagram for the case $i \notin F$.

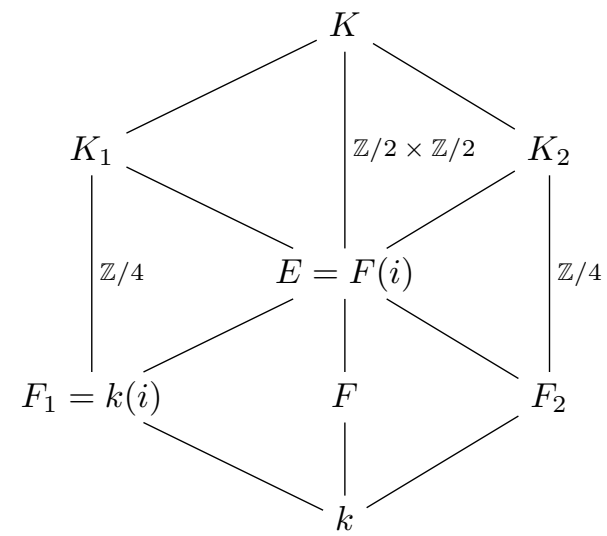

Fix any prime $v$ of degree 2 in $F / k$. We claim any prime $w$ of $K$ above $v$ has degree $\geq 4$ over $k$. If not, $v$ splits into 2 primes $v_{1}, v_{2}$ in $E$. Say $w \mid v_{1}$. By Ram15, Lemma 5.8], $v_{1}$ is degree 2 either over $F_{1}$ or over $F_{2}$. Let $i \in\{1,2\}$ be such that $v_{1}$ is degree 2 over $F_{i}$, and let $u$ be the prime of $F_{i}$ under $v_{i}$. Then $u$ is inert in $K_{i} / F_{i}$, and so $v_{1}$ is inert in $K_{i} / E$. Hence $w$ has degree $\geq 2$ in $K / E$, and therefore degree $\geq 4$ in $K / k$, as claimed.

Thus, for such $K, \mathcal{L}_{K}(s)$ is analytic in $\operatorname{Re}(s)>\frac{1}{4}$.

REMARK 2. The above argument for $p>2$ in fact shows the following: if $F / k$ is any extension, $\pi$ is tempered, and $\rho$ corresponds to $\pi$ at all but finitely many places of degree $\leq 2$, then $L^{*}(s, \rho)$ is entire.

COROLlary 3.2. With $K$ as in the previous proposition, and $\chi$ and idele class character of $K$. Then $L^{*}\left(s, \rho_{K} \otimes \chi\right)$ is entire.

Proof. First suppose $p>2$, so we can take $K=F$. Then if $\chi$ is an idele class character of $F, \rho \otimes \chi$ and $\pi \otimes \chi$ locally correspond at almost all degree 1 places, so the previous proposition again applies to say $L^{*}(s, \rho \otimes \chi)$ is entire.

Now suppose $p=2$, and let $K$ be an extension as constructed in the proof of the above proposition. Then, for all but finitely many places of degree $\leq 2$, we have that $\rho_{K}$ and $\pi_{K}$ locally correspond. Hence the same is true for $\rho_{K} \otimes \chi$ and $\pi_{K} \otimes \chi$ for an idele class character $\chi$ of $K$. The above remark then gives the corollary.

Corollary 3.3. With $K$ as in proposition above, the Artin representation $\rho_{K}$ is automorphic, and corresponds to the base change $\pi_{K}$. 
Proof. The automorphy of $\rho_{K}$ now follows from the previous corollary and the converse theorem (Theorem 1.4). Furthermore, since $\rho_{K}$ corresponds to an automorphic representation which agrees with $\pi_{K}$ at a set of places of density 1 , it must agree with $\pi_{K}$ everywhere by [Ram94].

\section{Descent}

In this section, we will finish the proof of Theorem $\mathrm{A}$, that $\rho$ corresponds to $\pi$ globally. The last corollary (together with Proposition 1.5) already tells us this is the case if $p>2$, so we may assume $p=2$ in what follows. In the previous section, we showed $\rho_{K}$ corresponds to $\pi_{K}$ over a suitable quadratic (when $i \in F$ ) or biquadratic (when $i \notin F$ ) extension $K / F$. Here we will show that by varying our choice of $K$, we can descend this correspondence to $F$.

As above, let $S_{2}$ be the set of finite unramified primes $v$ of degree 2 in $F / k$ at which $\rho_{v}$ and $\pi_{v}$ are both unramified but do not correspond. If $S_{2}$ is finite, then an argument of Deligne-Serre [DS74] (cf. Mar04, Appendix A] or Proposition 1.5) already tells us $\rho$ and $\pi$ correspond everywhere (over $F$ at finite places and at the archimedean place over $\mathbb{Q}$ ), so we may assume $S_{2}$ is infinite. As before, write $F$ as $k(\sqrt{\alpha})$ for some $\alpha \in k$.

First suppose that $F$ contains $i$. Then, as in the proof of Proposition 3.1 $K=k\left(\alpha^{1 / 4}\right)$ is a quadratic extension of $F$, with $K$ cyclic over $k$, and $\pi_{K}$ corresponds to $\rho_{K}$ globally. Let $T_{2}$ denote the subset of $S_{2}$ consisting of finite places $v$ of $F$ which are unramified in $K$. Clearly, the complement of $T_{2}$ in $S_{2}$ is finite.

We claim that $T_{2}$ is empty, so that $\rho$ and $\pi$ correspond everywhere, already over $F$. Suppose not. Pick any element $v_{0}$ of $T_{2}$. Since $\operatorname{det}(\rho)$ corresponds to $\omega_{\pi}$ at all places, and since $\rho_{K}$ and $\pi_{K}$ correspond exactly, if $\chi_{v_{0}}$ is the quadratic character of $F_{v_{0}}$ attached to the quadratic extension $K_{\bar{v}_{0}}, \rho_{v_{0}}$ must correspond to $\pi_{v_{0}} \otimes \chi_{v_{0}}$. As $K_{\bar{v}_{0}} / F_{v_{0}}$ is unramified, so is $\chi_{v_{0}}$.

Now we may modify the choice of $K$ as follows. Pick a $\beta$ in $k$ which is a square but not a fourth power, and put $\tilde{K}=k\left((\alpha \beta)^{1 / 4}\right)$. Then $\tilde{K}$ contains $F$ and is a cyclic quartic extension of $k$ (just like $K$ ), so that all but a finite number of places in $S_{2}$ are inert in $\tilde{K}$. But now we may choose $\beta$ such that $v_{0}$ ramifies in $\tilde{K}$. It follows (as above) that over $\tilde{K}$, the base changes of of $\pi$ and $\rho$ correspond everywhere, and that $\rho_{v_{0}}$ corresponds to $\pi_{v_{0}} \otimes \tilde{\chi}_{v_{0}}$, where $\tilde{\chi}_{v_{0}}$ is now the ramified local character at $v_{0}$ (attached to $\left.\tilde{K} / F\right)$. This gives a contradiction as we would then need $\pi_{v_{0}} \otimes \chi_{v_{0}}$ to be isomorphic to $\pi_{v_{0}} \otimes \tilde{\chi}_{v_{0}}$ (only the latter twist is ramified). So the only way to resolve this is to have $\rho_{v_{0}}$ correspond to $\pi_{v_{0}}$. Then $v_{0}$ cannot lie in $T_{2}$. As it was taken to be a general element of $T_{2}$, the whole set $T_{2}$ must be empty, proving the claim. We are now done if $i$ belongs to $F$.

Thus we may assume from here on that $i \notin F$. Then $E=F(i)$ is a biquadratic extension of $k$, and every element $v$ of $S_{2}$ which is unramified in $E$ must split there, say into $v_{1}$ and $v_{2}$. So it suffices to prove that $\rho$ and $\pi$ correspond exactly over $E$. Suppose not. Without loss of generality, say $\rho_{E, v_{1}}$ and $\pi_{E, v_{1}}$ do not correspond.

Consider the construction of $K$, biquadratic over $F$, with subfields $K_{1}, K_{2}, E$, $F_{1}, F_{2}$ as in the proof of Proposition 3.1. Interchanging indices on $K_{i}$ 's and $F_{i}$ 's if necessary, we may assume that $v_{1}$ has degree 2 in $E / F_{1}$, so it lies under a unique prime $w$ in $K_{1}=F_{1}\left(\alpha_{1}^{1 / 4}\right)$. Since $w$ splits in $K, \rho_{K_{1}, w}$ must correspond to $\pi_{K_{1}, w} \otimes \chi$, where $\chi$ is the unramified quadratic character attached to $K_{1, w} / E_{v_{1}}$. 
As in the previous case, we may modify $K_{1}$ to $\tilde{K}_{1}=F_{1}\left(\left(\alpha_{1} \beta_{1}\right)^{1 / 4}\right)$ for some

$\beta_{1} \in F_{1}$ which is a square but not a fourth power. We may choose $\beta_{1}$ so that $\tilde{K}_{1} / E$ is ramified. Then $\tilde{K}_{1} / F_{1}$ is a cyclic extension of degree 4 containing $E$, and Proposition 3.1 is still valid with $K$ replaced by $\tilde{K}=\tilde{K}_{1} K_{2}$. Let $\tilde{w}$ be the prime of $\tilde{K}_{1}$ over $v_{1}$. Since $\tilde{w}$ splits over $\tilde{K}$, we also have that $\rho_{\tilde{K}_{1}, w}$ corresponds to $\pi_{\tilde{K}_{1}, w} \otimes \tilde{\chi}$, where $\tilde{\chi}$ is the ramified quadratic character associated to $\tilde{K}_{1, w} / E_{v_{1}}$. Again, this implies $\pi_{K_{1}, w} \otimes \chi \simeq \pi_{\tilde{K}_{1}, w} \otimes \tilde{\chi}$, which is a contradiction.

\section{References}

[BB11] Valentin Blomer and Farrell Brumley, On the Ramanujan conjecture over number fields, Ann. of Math. (2) 174 (2011), no. 1, 581-605, DOI 10.4007/annals.2011.174.1.18. MR2811610

[BK11] Andrew R. Booker and M. Krishnamurthy, A strengthening of the GL(2) converse theorem, Compos. Math. 147 (2011), no. 3, 669-715, DOI 10.1112/S0010437X10005087. MR2801397 (2012f:11090)

[DS74] Pierre Deligne and Jean-Pierre Serre, Formes modulaires de poids 1, Ann. Sci. École Norm. Sup. (4) 7 (1974), 507-530 (1975) (French). MR0379379 (52 \#284)

[GJ78] Stephen Gelbart and Hervé Jacquet, A relation between automorphic representations of GL(2) and GL(3), Ann. Sci. École Norm. Sup. (4) 11 (1978), no. 4, 471-542. MR533066 (81e:10025)

[JL70] H Jacquet and R. P. Langlands, Automorphic forms on GL(2), Lecture Notes in Mathematics, vol. 114, Springer-Verlag, Berlin-New York, 1970. MR0401654 (70)

[JS85] Hervé Jacquet and Joseph Shalika, A lemma on highly ramified $\epsilon$-factors, Math. Ann. 271 (1985), no. 3, 319-332, DOI 10.1007/BF01456070. MR787183 (87i:22048)

[Kha10] Chandrashekhar Khare, Serre's conjecture and its consequences, Jpn. J. Math. 5 (2010), no. 1, 103-125, DOI 10.1007/s11537-010-0946-5. MR2609324 (2011d:11121)

[Kim03] Henry H. Kim, Functoriality for the exterior square of $\mathrm{GL}_{4}$ and the symmetric fourth of $\mathrm{GL}_{2}$, J. Amer. Math. Soc. 16 (2003), no. 1, 139-183, DOI 10.1090/S0894-0347-0200410-1. With appendix 1 by Dinakar Ramakrishnan and appendix 2 by Kim and Peter Sarnak. MR1937203 (2003k:11083)

[KS02] Henry H. Kim and Freydoon Shahidi, Cuspidality of symmetric powers with applications, Duke Math. J. 112 (2002), no. 1, 177-197, DOI 10.1215/S0012-9074-02-11215-0. MR1890650 (2003a:11057)

[Kut80] Philip Kutzko, The Langlands conjecture for $\mathrm{Gl}_{2}$ of a local field, Ann. of Math. (2) 112 (1980), no. 2, 381-412, DOI 10.2307/1971151. MR592296 (82e:12019)

[Lan80] Robert P. Langlands, Base change for GL(2), Annals of Mathematics Studies, vol. 96, Princeton University Press, Princeton, N.J.; University of Tokyo Press, Tokyo, 1980. MR574808 (82a:10032)

[LRS99] Wenzhi Luo, Zeév Rudnick, and Peter Sarnak, On the generalized Ramanujan conjecture for GL(n), Automorphic forms, automorphic representations, and arithmetic (Fort Worth, TX, 1996), Proc. Sympos. Pure Math., vol. 66, Amer. Math. Soc., Providence, RI, 1999, pp. 301-310. MR1703764 (2000e:11072)

[Mar04] Kimball Martin, Four-dimensional Galois representations of solvable type and automorphic forms, ProQuest LLC, Ann Arbor, MI, 2004. Thesis (Ph.D.)-California Institute of Technology. MR2706615

[Ram15] Dinakar Ramakrishnan, A mild Tchebotarev theorem for GL(n), J. Number Theory 146 (2015), 519-533, DOI 10.1016/j.jnt.2014.08.002. MR3267122

[Ram94] , A refinement of the strong multiplicity one theorem for GL(2). Appendix to: "l-adic representations associated to modular forms over imaginary quadratic fields. II" [Invent. Math. 116 (1994), no. 1-3, 619-643; MR1253207 (95h:11050a)] by R. Taylor, Invent. Math. 116 (1994), no. 1-3, 645-649, DOI 10.1007/BF01231576. MR1253208 (95h:11050b)

[RT83] J. D. Rogawski and J. B. Tunnell, On Artin L-functions associated to Hilbert modular forms of weight one, Invent. Math. 74 (1983), no. 1, 1-42, DOI 10.1007/BF01388529. MR722724 (85i:11044) 
[Wil88] A. Wiles, On ordinary $\lambda$-adic representations associated to modular forms, Invent. Math. 94 (1988), no. 3, 529-573, DOI 10.1007/BF01394275. MR969243 (89j:11051)

(Kimball Martin) Department of Mathematics, University of OkLahoma, Norman, OK 73019 USA

(Dinakar Ramakrishnan) Department of Mathematics, Caltech, Pasadena, CA 91125 